\title{
Myo-Myo: Yes, papa. Eating sugar? No, papa!
}

\author{
Modulating the myocardial menu for imaging coronary inflammation...
}

\author{
Jagat Narula $\cdot$ H. William Strauss
}

Published online: 27 September 2011

(C) Springer-Verlag 2011

Each year 295,000 hospital cardiac arrests occur in the USA [1], with a median survival rate of $7.9 \%$. Only $33 \%$ of these cardiac arrest patients have symptoms earlier than $1 \mathrm{~h}$ before death. These sobering statistics emphasize the urgent need to develop a reliable method to identify patients at high risk for a fatal cardiac event. Although primary prevention is a successful strategy in a population, fateful events continue to occur [2] in a sizable proportion of subjects treated with the best of medical regimens. To offer the most effective care may require identification and possibly local treatment of high-risk coronary lesions in each patient.

High-risk plaque includes a voluminous necrotic core, containing lipids, oxidized lipids, numerous dead cells, and activated macrophages $[3,4]$. The necrotic core is covered by an attenuated and an inflamed fibrous cap. Identifying the thin cap is only possible by invasive intravascular imaging. The necrotic core, however, can be assessed by computed tomography angiography $[5,6]$. The necrotic core appears as a low-attenuation or low-density area on $\mathrm{CT}$ and often induces expansive (or outward) remodeling of the affected vascular segment. On the other hand, inflammation may be amenable to molecular imaging strategies $[7,8]$. Since the intense metabolic demand of the lesional macrophages is met by exogenous glucose diffusing into the lesion from the blood, it has been proposed that positron-labeled fluorodeoxyglucose (FDG)

This Editorial Commentary refers to the article http://dx.doi.org/ 10.1007/s00259-011-1873-2

J. Narula $\cdot$ H. W. Strauss $(\bowtie)$

Mount Sinai Medical Center \& Memorial

Sloan-Kettering Cancer Center,

New York, NY, USA

e-mail: bill.strauss@strausspartners.com should concentrate at the site of inflammation, allowing visualization on positron emission tomography (PET) images [9].

FDG PET/CT images in cancer patients often show multiple focal sites of uptake in the aorta [10]. In patients undergoing carotid endarterectomy, FDG PET scans confirmed that focal tracer uptake in the carotid arteries of humans is due to localization in macrophages [11]. In prospective investigations, FDG uptake occurred selectively in symptomatic carotid vascular disease [12] and was significantly reduced in response to statin treatment [13]. On the other hand, FDG uptake is seldom seen in the coronary vasculature $[10,14]$. There are several factors limiting visualization of lesions in the coronary arteries including: physiologic FDG uptake in the myocardium, limiting the contrast in the lesion due to high background; small size of coronary lesions (often less than $2 \mathrm{~mm}^{2}$ ), which requires resolution recovery for reliable quantitation of FDG uptake in the vessels; and blurring due to cardiac and respiratory motion during the acquisition $[15,16]$.

Under normal aerobic conditions fatty acid is the preferred metabolic substrate for the myocardium. Cardiomyocytes, however, are omnivorous, and can consume almost all alternative substrates, including glucose, lactate, pyruvate, and even ketones [17] (Fig. 1). Glucose serves as the preferred substrate for oxidative metabolism postprandially, while fatty acids are consumed preferentially in fasting states. It is logical that fasting may substantially suppress myocardial FDG uptake. Experience has demonstrated that fasting up to $18 \mathrm{~h}$ substantially increases fatty acid uptake and metabolism in the myocardium $[18,19]$; it does not reliably eliminate glucose consumption.

From a clinical perspective, it is easier to increase myocardial glucose uptake than suppress it. Although 
Fig. 1 Schematic illustration to discuss strategies for restricting myocardial glucose uptake. Restricting the glucose and feeding high-fat diet increases the free fatty acid (FFA) consumption by myocardium (green pathways). FFA delivery and acetyl coenzyme A (CoA) excess in mitochondria decrease production and

consumption of pyruvate in the tricarboxylic acid (TCA) cycle, by direct or indirect suppression of pyruvate dehydrogenase (PDH), phosphofructokinase (PFK), and hexokinase (HK). The insulin-based glucose transport is also decreased. Alternatively, calcium channel blockers may restrict glucose entry into the myocytes and glycogenolysis (red pathways). On the other hand, shift from FFA to glucose consumption may be induced through activation of PDH and pyruvate utilization (e.g., dichloroacetate), restricting FFA uptake either by inhibition of CPT I/II (e.g., perhexiline and etomoxir) or forcing additional glucose uptake (e.g., glucose-insulin infusion), and restriction of FFA beta-oxidation (e.g., trimetazidine and ranolazine) (asterisks)

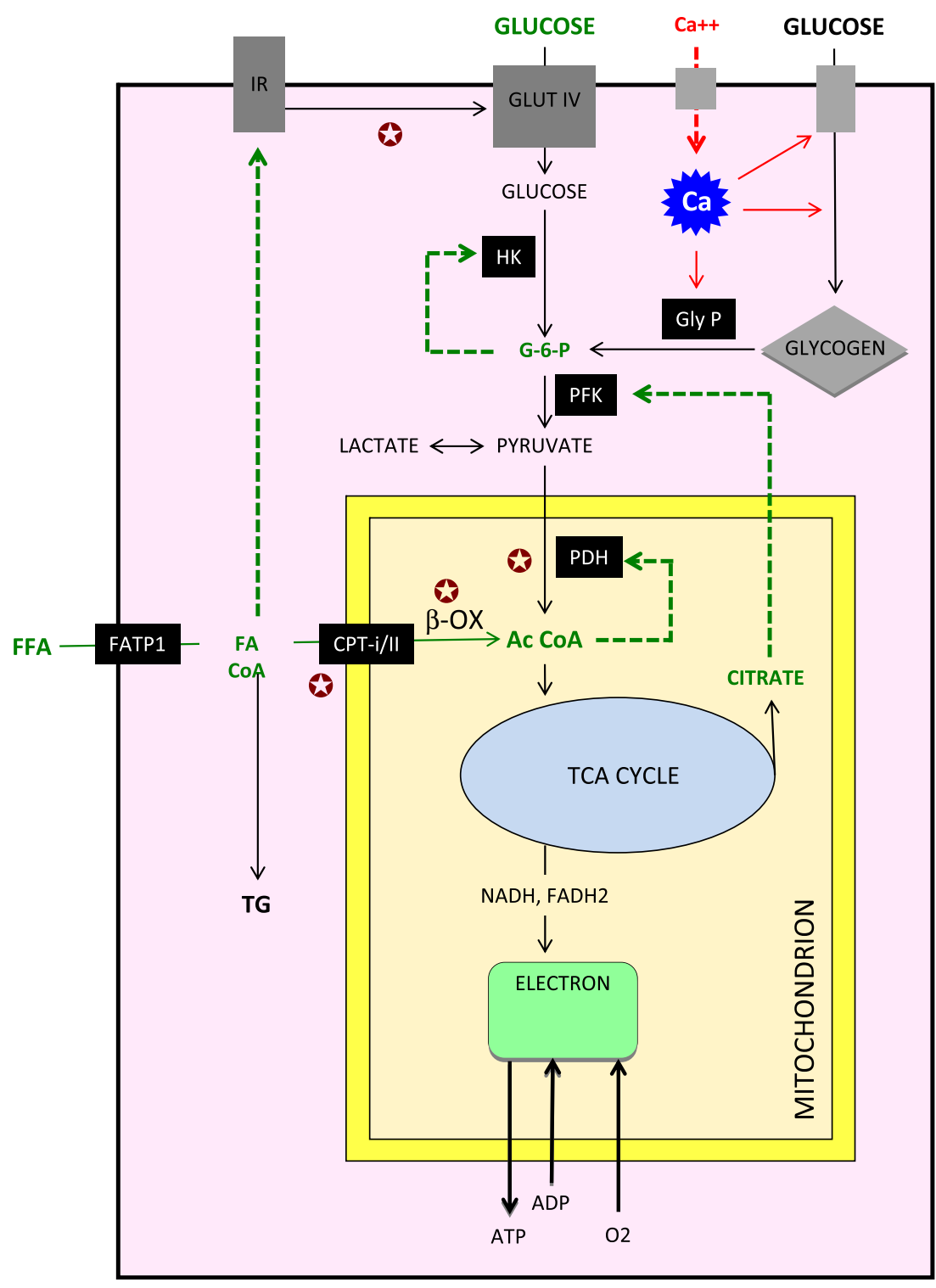

glucose oxidation, compared to fatty acid oxidation, yields less ATP per molecule of $\mathrm{CO}_{2}$ produced, it produces $15 \%$ more ATP per unit oxygen consumption [20]. Glucose consumption can be increased in patients by infusion of glucose, insulin, and potassium. Mitochondrial glucose metabolism has been effectively increased with agents that activate pyruvate dehydrogenase to increase oxidation of pyruvate (such as dichloroacetate), increase insulin sensitivity (such as the thiazolidinediones), inhibit mitochondrial fatty acid uptake through suppression of carnitine palmitoyltransferase (CPT) I or II [such as perhexiline, etomoxir, and glucagon-like peptide (GLP-1)], or inhibit the enzyme long-chain 3-ketoacyl coenzyme A thiolase to increase pyruvate $\beta$-oxidation and reduce lactate accumulation (such as trimetazidine and ranolazine) [20].

Unlike tweaking mitochondrial mechanisms for increased glucose consumption, facilitation of fatty acid metabolism can be better achieved by reducing glucose uptake and/or increasing fatty acid availability at the myocellular level. Such a metabolic switch can be achieved by feeding a high-fat, low-carbohydrate meal for a whole day before the scan and an oil drink just before the test [21]. Whereas glucose availability promotes glucose oxidation, increased free fatty acid (FFA) availability promotes FFA oxidation. However, given the ubiquity of glucose consumption even in the fatty acid excess state, the glucose exclusion is partial at best. Especially since comorbidities associated with high adrenergic state, such as hypertension and heart failure, promote glucose uptake even during circulating FFA excess. Wykrzykowska et al. [22] retrospectively analyzed a group of patients undergoing FDG PET/CT study for the assessment of malignancy. As a part of the protocol, these patients had received a low-carbohydrate, high-fat meal the night before 
and a vegetable oil drink on the morning of the study to evaluate the adequacy of myocardial uptake suppression. In two thirds of their patients, myocardial suppression was considered adequate; failure of suppression was attributed to dietary noncompliance. Against the lower myocardial background activity, coronary FDG uptake could be detected in up to one half of their patients in one or more vascular segments. Rogers and colleagues [23] used this lead for a prospective study to assess the feasibility of myocardial suppression of FDG uptake for imaging inflammation in proximal coronary vasculature. Further, they only included patients who had undergone a coronary interventional procedure with a stent placement for an acute coronary event or stable coronary disease with the past 1 week, so that they could exploit the stent landmark in simultaneously performed CT angiogram as the site of tracer uptake. Distinct FDG uptake was observed in the stented culprit lesions of the acute coronary syndrome (ACS) patients. The stents placed in patients with stable disease did not accumulate FDG, suggesting lack of inflammation in chronic stable disease. FDG uptake was spontaneously seen in the aorta and left main trunk of a subset of the patients and the magnitude of FDG uptake in stented or spontaneous sites correlated with the circulating Creactive protein (CRP) levels. Although these two studies have offered promising results, suppression of myocardial glucose activity was often incomplete.

The manuscript by Gaeta et al. [24] in this issue of the European Journal of Nuclear Medicine and Molecular Imaging proposes the use of a calcium channel blocker (verapamil) to inhibit myocardial glucose uptake. This approach may be used independently or in association with dietary manipulation. Glucose uptake in the myocardium is regulated by catecholamines, insulin, glucagon, and thyroid hormones. It is also facilitated by intracellular calcium. Therefore, two agents used commonly in patients with coronary disease, beta-adrenergic and calcium channel blockers, intuitively constitute attractive interventions for restricting myocardial glucose uptake. Use of both agents, which also cause negative chronotropic effects, may help improve image quality when CT angiogram is simultaneously acquired. Although betablockers seem to be an obvious choice to restrict insulinsensitive glucose transporter 4 (GLUT4)-related transport, they have also been demonstrated to paradoxically decrease FFA oxidation in favor of glucose oxidation and increased FDG uptake. On the other hand, $\mathrm{Ca}^{2+}$-dependent intracellular adenosine 3',5'-monophosphate (cAMP) upregulation facilitates intramyocardial glucose transport by sarcolemmal recruitment of glucose transporters [25, 26] and may also stimulate glycogen breakdown (via phosphorylase activation) and glucose consumption, with consequent increase in myocardial glucose uptake.
Although Gaeta et al. only studied ten mice the manuscript convincingly demonstrates reduction in myocardial glucose uptake. The dose dependence, which would have been expected, was not observed, possibly due to the small number of animals. Although there was a substantial variation in myocardial FDG uptake among mice of similar age and sex, the reduction in glucose uptake was reassuringly consistent. If confirmed in a larger number of preclinical or clinical experiments, it may obviate the need for a dietary protocol, such as employed by Rogers et al. [23]. It also remains to be seen if diltiazem would influence the myocardial glucose uptake similarly. Calcium signaling plays an important role in the activity of monocyte-macrophages and it will need to be proven that glucose uptake of these cells is not minimized by calcium blocker administration. Analyzing experimental evidence for dietary modification, Fox and Strauss commented that tumor FDG uptake was not altered [15], nor was the FDG uptake affected at nonvascular inflammatory sites by the high-fat, lowcarbohydrate diet preparation [22]. Since the plaque microenvironment is hypoxic, cells in the lesion should favor a glycolytic state over oxidative metabolism even in the presence of an altered nutrient environment $[15,27]$.

Gaeta and colleagues [24] are to be congratulated for this proof of principle study. We await their definitive clinical study with a focus on the detection of coronary inflammation.

\section{References}

1. Roger VL, Go AS, Lloyd-Jones DM, Adams RJ, Berry JD, Brown TM, et al. Heart disease and stroke statistics-2011 update: a report from the American Heart Association. Circulation 2011;123:e18-e209.

2. Wiviott SD, Cannon CP, Morrow DA, Ray KK, Pfeffer MA, Braunwald E, et al. Can low-density lipoprotein be too low? The safety and efficacy of achieving very low low-density lipoprotein with intensive statin therapy: a PROVE IT-TIMI 22 substudy. J Am Coll Cardiol 2005;46:1411-6. Erratum in: J Am Coll Cardiol 2006;47:472.

3. Burke AP, Farb A, Malcom GT, Liang YH, Smialek J, Virmani R. Coronary risk factors and plaque morphology in men with coronary disease who died suddenly. N Engl J Med 1997;336:1276-82.

4. Narula J, Strauss HW. The popcorn plaques. Nat Med 2007;13:532-4.

5. Motoyama S, Kondo T, Sarai M, Sugiura A, Harigaya H, Sato T, et al. Multislice computed tomographic characteristics of coronary lesions in acute coronary syndromes. J Am Coll Cardiol 2007;50:319-26.

6. Motoyama S, Sarai M, Harigaya H, Anno H, Inoue K, Hara $\mathrm{T}$, et al. Computed tomographic angiography characteristics of atherosclerotic plaques subsequently resulting in acute coronary syndrome. J Am Coll Cardiol 2009;54:49-57.

7. Narula J, Garg P, Achenbach S, Motoyama S, Virmani R, Strauss HW. Arithmetic of vulnerable plaques for noninvasive imaging. Nat Clin Pract Cardiovasc Med 2008;5(Suppl 2):S2-10. 
8. Tahara N, Imaizumi T, Virmani R, Narula J. Clinical feasibility of molecular imaging of plaque inflammation in atherosclerosis. $\mathrm{J}$ Nucl Med 2009;50:331-4.

9. Vallabhajosula S, Fuster V. Atherosclerosis: imaging techniques and the evolving role of nuclear medicine. J Nucl Med 1997;38:1788-96.

10. Dunphy MP, Freiman A, Larson SM, Strauss HW. Association of vascular 18F-FDG uptake with vascular calcification. J Nucl Med 2005;46:1278-84.

11. Tawakol A, Migrino RQ, Bashian GG, Bedri S, Vermylen D, Cury $\mathrm{RC}$, et al. In vivo $18 \mathrm{~F}$-fluorodeoxyglucose positron emission tomography imaging provides a noninvasive measure of carotid plaque inflammation in patients. J Am Coll Cardiol 2006;48:1818-24.

12. Rudd JH, Warburton EA, Fryer TD, Jones HA, Clark JC, Antoun $\mathrm{N}$, et al. Imaging atherosclerotic plaque inflammation with [18F]fluorodeoxyglucose positron emission tomography. Circulation 2002;105:2708-11.

13. Tahara N, Kai H, Ishibashi M, Nakaura H, Kaida H, Baba K, et al. Simvastatin attenuates plaque inflammation: evaluation by fluorodeoxyglucose positron emission tomography. J Am Coll Cardiol 2006;48:1825-31.

14. Alexanderson E, Slomka P, Cheng V, Meave A, Saldaña Y, García-Rojas L, et al. Fusion of positron emission tomography and coronary computed tomographic angiography identifies fluorine 18 fluorodeoxyglucose uptake in the left main coronary artery soft plaque. J Nucl Cardiol 2008;15:841-3.

15. Fox JJ, Strauss HW. One step closer to imaging vulnerable plaque in the coronary arteries. J Nucl Med 2009;50:497-500.

16. Narula J, Chandrashekhar Y. Molecular imaging of coronary inflammation: overcoming hurdles one at a time.... JACC Cardiovasc Imaging 2010;3:448-50.

17. Bing RJ, Fenton JC. Cardiac metabolism. Annu Rev Med $1965 ; 16: 1-2$.

18. de Groot M, Meeuwis AP, Kok PJ, Corstens FH, Oyen WJ. Influence of blood glucose level, age and fasting period on non- pathological FDG uptake in heart and gut. Eur J Nucl Med Mol Imaging 2005;32:98-101.

19. Shreve PD, Anzai Y, Wahl RL. Pitfalls in oncologic diagnosis with FDG PET imaging: physiologic and benign variants. Radiographics 1999;19:61-77.

20. Morrow D, Givertz MM. Modulation of myocardial energetics: emerging evidence for a therapeutic target in cardiovascular disease. Circulation 2005;112:3218-21.

21. Williams G, Kolodny GM. Suppression of myocardial 18F-FDG uptake by preparing patients with a high-fat, low-carbohydrate diet. AJR Am J Roentgenol 2008;190:W151-6.

22. Wykrzykowska J, Lehman S, Williams G, Parker JA, Palmer MR, Varkey S, et al. Imaging of inflamed and vulnerable plaque in coronary arteries with $18 \mathrm{~F}-\mathrm{FDG}$ PET/CT in patients with suppression of myocardial uptake using a low-carbohydrate, high-fat preparation. J Nucl Med 2009;50:563-8.

23. Rogers IS, Nasir K, Figueroa AL, Cury RC, Hoffmann U, Vermylen DA, et al. Feasibility of FDG imaging of the coronary arteries: comparison between acute coronary syndrome and stable angina. JACC Cardiovasc Imaging 2010;3:388-97.

24. Gaeta C, Fernández Y, Pavía J, Flotats A, Artigas C, Deportos J, et al. Reduced myocardial (18)F-FDG uptake after calcium channel blocker administration. Initial observation for a potential new method to improve plaque detection. Eur J Nucl Med Mol Imaging 2011.

25. Depre C, Ponchaut S, Deprez J, Maisin L, Hue L. Cyclic AMP suppresses the inhibition of glycolysis by alternative oxidizable substrates in the heart. J Clin Invest 1998;101:390-7.

26. Dobson Jr JG, Ross Jr J, Mayer SE. The role of cyclic adenosine $3^{\prime}, 5^{\prime}$-monophosphate and calcium in the regulation of contractility and glycogen phosphorylase activity in guinea pig papillary muscle. Circ Res 1976;39:388-95.

27. Nuutinen J, Minn H, Bergman J, Haaparanta M, Ruotasalainen U, Laine $\mathrm{H}$, et al. Uncoupling of fatty acid and glucose metabolism in malignant lymphoma: a PET study. Br J Cancer 1999;80:513-8. 